\title{
Development of a Proton Computed Tomography System for Pre-Clinical Tests
}

\author{
C. Civinini, M. Brianzi, M. Bruzzi, M. Bucciolini, M. Carpinelli, G. A. P. Cirrone, G. Cuttone, D. Lo Presti, S. \\ Pallotta, C. Pugliatti, N. Randazzo, F. Romano, M. Scaringella, V. Sipala, C. Stancampiano, C. Talamonti, M. Tesi, \\ E. Vanzi, M. Zani
}

\begin{abstract}
Proton Computed Tomography (pCT) is an imaging method under investigation with the aim to improve the accuracy of treatment planning and patient positioning in hadron therapy. A pCT system should be capable to measure tissue electron density with an accuracy better than $1 \%$ and with a spatial resolution better than $1 \mathrm{~mm}$. A first apparatus based on a silicon tracker and a YAG:Ce crystal calorimeter, with a $5 \times 5 \mathrm{~cm}^{2}$ active area, has been already developed by the authors in the framework of the PRIMA (Proton Imaging) collaboration and tested with proton beams with promising results. A second prototype with larger area $\left(5 \times 20 \mathrm{~cm}^{2}\right)$ and improved data acquisition system is being designed. The details about this new prototype will be presented in this work.
\end{abstract}

\section{INTRODUCTION}

$\mathrm{T}$ HE main advantage of hadron therapy in cancer treatment, if compared with conventional $\mathrm{X}$-ray radiotherapy, is the high spatial conformity of the delivered dose to the target volume thanks to the characteristic energy loss of heavy charged particles as function of their penetration depth in matter. The maximum dose in a charged hadron path is released towards the end of its range (Bragg peak) so the delivered dose can be located at the desired depth by tuning the initial beam energy,

Manuscript received November 16, 2012. This work was supported by INFN CSN5 PRIMA+ experiment.

C. Civinini is with INFN - Florence Division, Florence, Italy (e-mail: civinini@fi.infn.it).

M. Brianzi is with INFN - Florence Division, Florence, Italy.

M. Bruzzi is with Energetics Department, University of Florence and INFN - Florence Division, Florence, Italy. Mara Bruzzi is spokesperson of the experiment.

M. Bucciolini, S. Pallotta, C. Talamonti and M. Zani are with Clinical Physiopathology Department, University of Florence and INFN - Florence Division, Florence, Italy and SOD Fisica Medica, Azienda OspedalieroUniversitaria Careggi, Florence, Italy.

M. Carpinelli and V. Sipala are with Chemistry and Pharmacy Department, University of Sassari, Sassari, Italy and INFN - Cagliari Division.

G. A. P. Cirrone, G. Cuttone and C. Stancampiano are with INFN Laboratori Nazionali del Sud, Catania, Italy.

D. Lo Presti, C. Pugliatti are with Physics Department, University of Catania and INFN - Catania Division, Catania, Italy.

N. Randazzo is with INFN - Catania Division, Catania, Italy.

F. Romano is with Centro Studi e Ricerche e Museo Storico della Fisica, Rome, Italy and INFN - Laboratori Nazionali del Sud, Catania, Italy.

M. Scaringella and M. Tesi are with Energetics Departments, University of Florence, Italy.

E. Vanzi is with SOD Fisica Medica, Azienda Ospedaliero-Universitaria Careggi, Florence, Italy. minimizing the dose to the healthy tissue surrounding the tumor. One of the main limitations in the precision that can be reached with hadron therapy is related to the poor accuracy in the stopping power (SP) distribution measurements which are required for treatment planning. Those maps are nowadays indirectly derived from X-ray CT [1]. The fundamental difference in the interaction with matter between photons and charged hadrons results in an error in the SP distribution measurement that limits the precision in the hadron range determination.

This uncertainty could be reduced by directly measuring the SP map using a proton beam with kinetic energy sufficient to cross the patient body. The proton detector system should be designed to partially overcome the limitations introduced by the intrinsic effect of the Multiple Coulomb Scattering (MCS). Typical crossed thicknesses could as thick as $20 \mathrm{~cm}$ water equivalent: in this case a $200 \mathrm{MeV}$ kinetic energy proton undergoes to an r.m.s. MCS angle of about 40mrad which corresponds to an r.m.s. of the projected displacement of about $3.2 \mathrm{~mm}$ [2]. With this numbers it is evident that simple proton projection radiographies have a too limited spatial resolution to be used to improve the SP map measurement. A possible solution is to measure the trajectory parameters of each proton both upstream and downstream the target together with the residual proton kinetic energy. With this information the so called proton 'Most Likely Path' (MLP) could be estimated [3]. It has been shown that the one sigma envelope of a transverse coordinate of the MLP of a $200 \mathrm{MeV}$ kinetic energy proton in $20 \mathrm{~cm}$ of water is of the order of $0.6 \mathrm{~mm} \mathrm{[3].}$ Assigning the proton energy loss to each MLP the 3dimensional SP map can be constructed, using appropriate reconstructing algorithms [4]. First measurements proved the potential of this approach [5-7].

A first pCT prototype, based on a silicon tracker and a YAG:Ce calorimeter with a $5 \times 5 \mathrm{~cm}^{2}$ active area and a $10 \mathrm{kHz}$ data rate, has been developed within the PRIMA collaboration [8-11]. First results on tomographic reconstruction show that a spatial resolution of the order of $1 \mathrm{~mm}$ can be achieved [1214]. A discussion of data analysis of recent test beams with high and low energy proton beams is given in [15-18].

An upgrade of the prototype, using the same detector technologies but with an increased active area $\left(5 \times 20 \mathrm{~cm}^{2}\right)$ and rate capability is under development. The choice of the rectangular geometry was made in view of using the instrument to perform imaging of objects with a size of interest 
for the application (e.g. a human head) by dividing the phantom in slices. To perform a pCT measurement in a reasonable amount of time, the DAQ system should be able to readout the experimental apparatus at a rate of the order of $1 \mathrm{MHz}$. In fact one single projection to be used to reconstruct a tomography image should be taken with a number of protons of the order of $10^{6}$. This number has to be multiplied by a factor of the order of 36-72 to uniformly cover the entire $360^{\circ}$ ending to a total acquisition time of the order of one minute.

The description of the new prototype will be given in the following sections.

\section{ARCHITECTURE}

The pCT system is made by a silicon microstrip tracker followed by a YAG:Ce calorimeter.

The tracker is made by four detection planes: two upstream the phantom and two downstream. Each plane measures a three dimensional trajectory impact point in order to eventually reconstruct two track segments: one upstream and the other downstream the object under test.

The calorimeter, totally absorbing the proton, gives an estimation of its residual energy and produces a trigger signal to be used to start the readout the different apparatus' components. A sketch of the pCT apparatus described in this note is reported in Fig. 1.

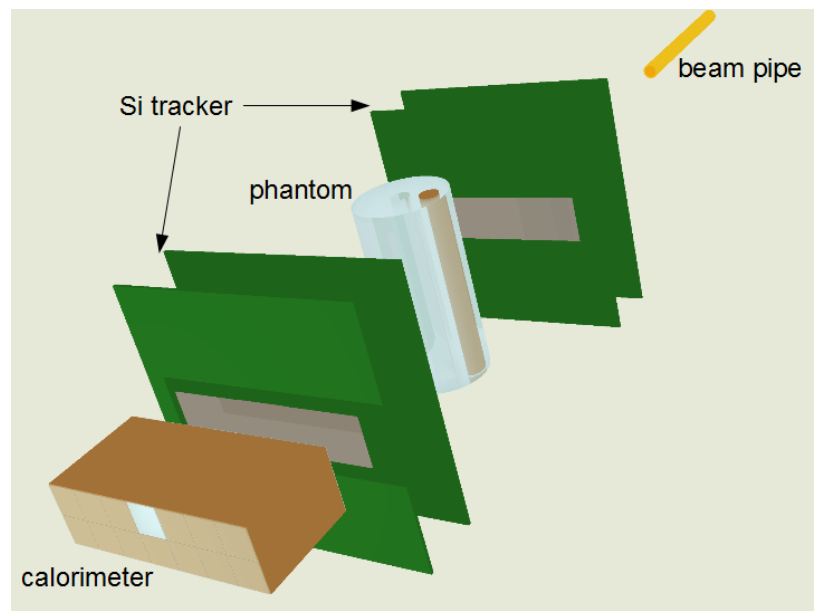

Fig. 1 layout of the pCT apparatus: two couple of silicon microstrip planes followed by a calorimeter. The proton beam illuminates the phantom which is rotated to take the projections needed to reconstruct its tomographic image.

\section{TRACKER}

\section{A. Detector plane}

Each tracker detector plane is realized using a single Printed Circuit Board (PCB) which hosts the silicon microstrip sensors, their front-end electronics together with FPGA implemented logic circuits for data reduction and transmission.

The silicon microstrip sensors are glued on a central aperture on the PCB. Four sensors are glued on a row on a side of the PCB, other fours are glued on the other side. The sensors on one side measure the $\mathrm{X}$ coordinate of the track impact point, the sensors on the opposite side, having the strips orthogonal to the others, measure the Y coordinate.

\section{B. Silicon sensors}

The silicon microstrip sensors used to assemble a tracker plane are single-sided devices of p-on-n type with a substrate $<100>$ orientation, a strip pitch of $200 \mu \mathrm{m}$ and a total depletion voltage equal or less than $85 \mathrm{~V}$. The total number of strips per sensor is 256 in an active area of $51 \times 50.66 \mathrm{~mm}^{2}$. The sensor thickness chosen for the tracker is discussed in section III.C.

\section{Silicon microstrip sensor assembly}

In order to avoid annular artifacts in FBP images, the detector it is important to build a tracker which is as much as possible hermetic, avoiding not only disconnected strips but also inactive areas due to the sensor assembly geometry.

The silicon microstrip sensors used in this project have an inactive zone of about $1 \mathrm{~mm}$. This is peripherally located between their sensitive area and external edges and hosts bias and guard rings together with cutting edge areas; these structures avoid excessive leakage current and possible early electrical breakdown.

A simple assembly of sensors glued side-by-side will lead to inactive areas which, in this case, could amount up to $4 \%$ of the total surface.

A hermetic tracker of $5 \times 20 \mathrm{~cm}^{2}$ total active area can be assembled overlapping the sensors by few millimeters to cover their inactive area.

The four sensors of the X-side should be additionally shifted by $1.75 \mathrm{~mm}$ in the direction parallel to their strips to allow the micro-bonding of the last 5 strips which otherwise would have their bonding pads inaccessible.

The four sensors of the Y-side should be overlapped by $2.44 \mathrm{~mm}$ or $2.24 \mathrm{~mm}$ (depending on which side of the device is overlapped: the one with or without bias resistors) to eliminate the inactive space.

\section{Silicon sensors thickness}

Due to the complexity of the system described in the present note, some aspects of the project require a careful choice of parameters to mitigate possible problems that can jeopardize the global performance of the entire pCT apparatus.

One of these choices is related to the silicon sensors' thickness. To reduce the multiple scattering influences on the reconstructed proton tracks the previous prototype makes use of $200 \mu \mathrm{m}$ thick silicon microstrip sensors; but, in that case, the readout strips were shorter than the ones foreseen in the $\mathrm{Y}$-side of the new tracker. This implies a bigger noise contribution, coming from the increased detector capacitance, which could be balanced by an increased charge released in a thicker, for example a $320 \mu \mathrm{m}$ thick, sensor.

The determination of this parameter is not an a priori problem free choice. In fact a greater material budget of the tracker in principle affects the detector performance altering the measured track parameters because of the multiple scattering in the sensors themselves. 
There is no doubt that this effect is always present if the sensors' thickness is increased: of immediate interest for this new pCT system is to quantify this degradation.

In the following the impact of the sensors' thickness on the measurement of $200 \mathrm{MeV}$ kinetic energy protons MLP in a 20 $\mathrm{cm}$ water equivalent phantom will be analyzed.

The MLP concept has been introduced by Williams [3] and generalized by Schulte et al. [19]. Given eight constraints at the boundaries of the phantom (2D-point and direction, both at entrance and exit of the phantom) is it possible to obtain an analytical expression of the most probable trajectory in a uniform medium.

An example of a MLP for a $200 \mathrm{MeV}$ proton in $20 \mathrm{~cm}$ of water is shown, together their one and two sigma error envelopes, in Fig. 2.

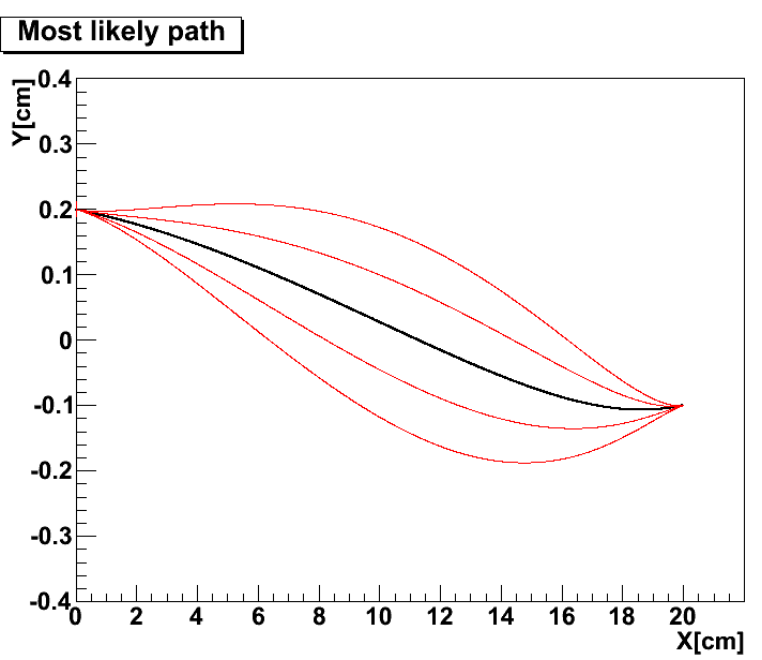

Fig. 2 MLP, black curve, of a $200 \mathrm{MeV}$ kinetic energy proton in $20 \mathrm{~cm}$ of water; entrance point and direction $(0.2 \mathrm{~cm}, 10 \mathrm{mrad})$, exit point and direction $(-0.1 \mathrm{~cm}, 10 \mathrm{mrad})$. In red the one and two sigma error envelopes due to MCS in the phantom are shown.

To evaluate the error contribution to the MLP envelope due to the multiple scattering generated by the sensors' material and the one due to the finite error on the position measurement by the sensors themselves, the error propagation to the MLP formula has been calculated. These contributions have been added in quadrature to the MLP envelope because, in a very good approximation, they are uncorrelated with respect to the MLP errors.

TABLE I. MULTIPLE SCATTERING CONTRIBUTION TO THE ERROR OF THE PROTON DIRECTION MEASUREMENT

\begin{tabular}{cll} 
& Silicon $200 \mu \mathrm{m}$ & Silicon $320 \mu \mathrm{m}$ \\
\hline $200 \mathrm{MeV}$ (entrance) & $1.9 \mathrm{mrad}$ & $2.5 \mathrm{mrad}$ \\
$90 \mathrm{MeV}$ (exit) & $4.1 \mathrm{mrad}$ & $5.2 \mathrm{mrad}$
\end{tabular}

The error on the position measurement has been assumed to be equal to the strip pitch divided by $\sqrt{ } 12$ on both side of the trajectory (in the present case $58 \mu \mathrm{m}$ ), while the error on the direction measurement has been calculated using the multiple scattering formula [20] and depends on the proton energy and detector thickness (Table 1).

The errors reported on Table 1 should be increased by the errors due to the trajectory angle measurements before and after the entrance and exit tracker planes. These errors are of the order of $0.5 \mathrm{mrad}$ for distances between the tracker planes greater than $15 \mathrm{~cm}$ and could then be neglected.

The total MLP error envelope could then be obtained adding in quadrature the different contributions: MLP error due to the multiple scattering in the phantom, silicon detector position and silicon multiple scattering errors (Fig. 3).

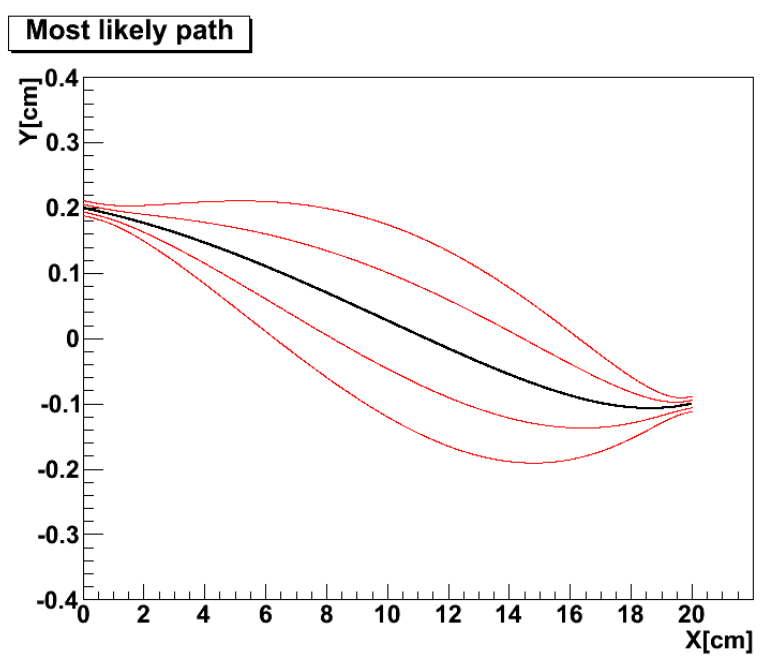

Fig. 3 MLP total envelope error for a proton with the same parameters as Fig. 2. The other error contributions have been calculated for a microstrip sensor pitch of $200 \mu \mathrm{m}$ and a thickness of $320 \mu \mathrm{m}$.

The different absolute error contributions to the total MLP envelope are plotted in Fig. 4.

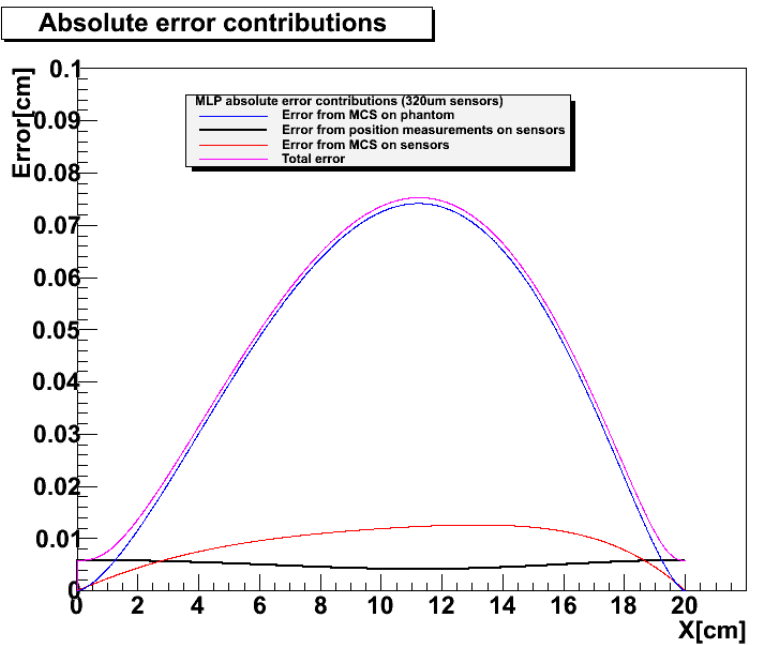

Fig. 4 Absolute error contribution to the MLP total envelope (same proton and detector parameters as Fig. 3).

The multiple scattering in the sensors is negligible if compared to the multiple scattering in the phantom, while the error due to the position measurement has some influence only 
in the regions close to the detector planes where the phantom multiple scattering is still low.

With this analysis it is possible to conclude that the use of thicker silicon microstrip sensors has a marginal contribution to the total error on reconstructed MLP and could be then be safely used to increase the noise margin in the electronic channel connected to the tracker's longer strips.

Also the limited overlap regions described in section III.B do not produce a substantial increase on the MLP total envelope error and could be adopted to eliminate the annular artifacts which are generated by large inactive detector regions.

\section{E. Front end read-out chip}

A new front-end readout chip, designed in the Austria Micro System $0.35 \mu \mathrm{m}$ technology, for the new microstrip sensors' configuration has been developed starting from results obtained from the previous prototype [5-7].

As in the previous prototype the chip consists of 32 independent channels, each including a charge sensitive preamplifier, a shaper and a comparator, suitable to produce a binary output using a fixed threshold. Moreover, in order to calibrate the device, two test input pads (one for even channels, the other for odd channels) are implemented to inject a known charge through an integrated capacitor in the input stage of the channels.

In the previous front-end chip version, the threshold was common to all channels. In this case a non-uniformity of channels response was observed: a standard deviation of about $2.5 \%$ on output pulse duration at fixed threshold has been measured [5]. Moreover a temperature dependency of the effective threshold value were found.

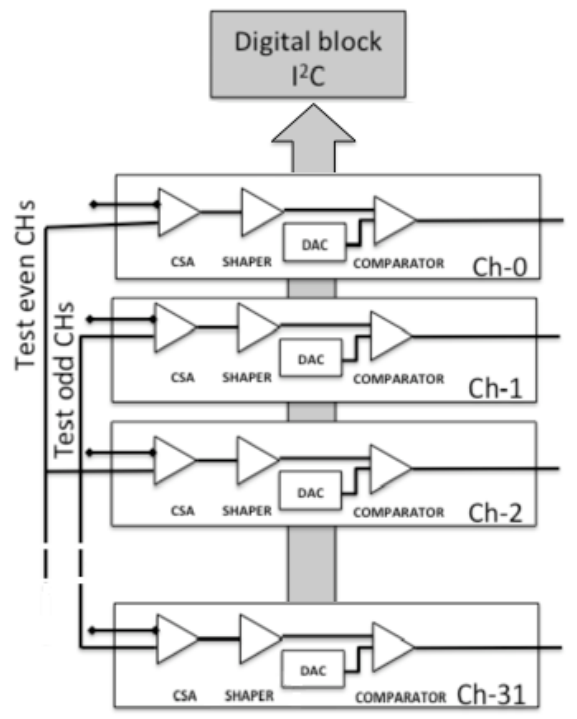

Fig. 5 Block diagram of the tracker front-end chip: each of the 32-channels is made by a Charge Sensitive Amplifier (CSA), a shaper, a discriminator and a DAC. An $\mathrm{I}^{2} \mathrm{C}$ digital block has been implemented to set DACs values.

In the new front-end chip each channel includes an 8-bit DAC for the generation of the threshold voltage. The DACs values are initially set using an $\mathrm{I}^{2} \mathrm{C}$ interface implemented on the chip. In Fig. 5 the scheme of the new chip is shown.

Additionally the new front-end chip has optimized performances in terms of noise and threshold dependence on temperature.

\section{F. Detector board}

The silicon microstrip detectors and front end read-out electronics of each $\mathrm{x}-\mathrm{y}$ plane will be mounted on a double sided PCB. The layout of the PCB is described in Fig. 6.
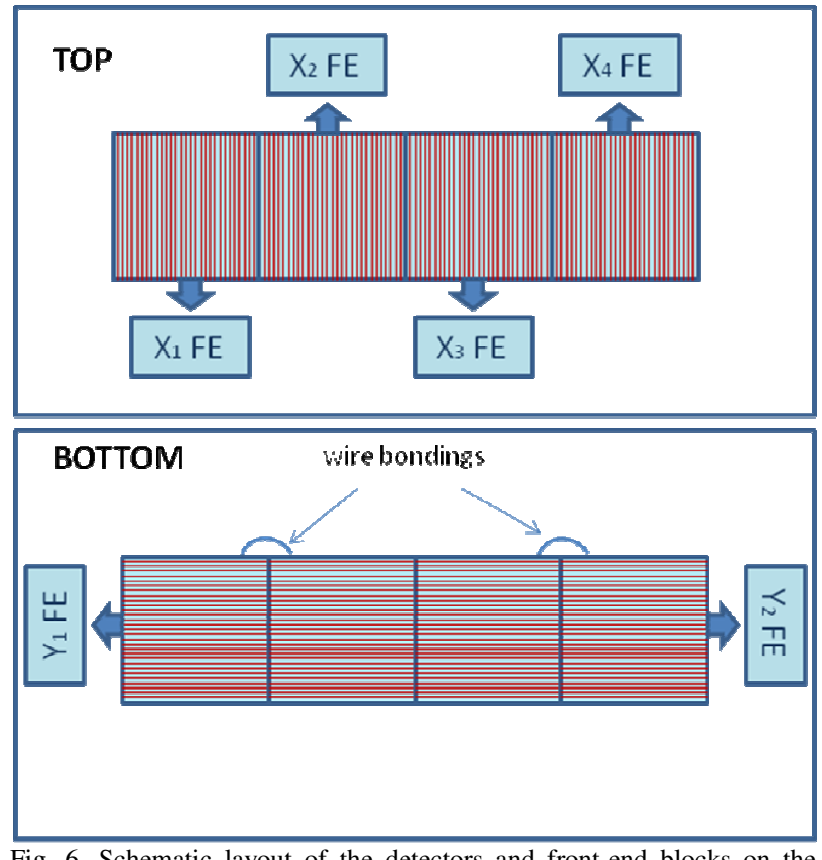
PCB.

The silicon microstrips on the top layer, measuring the $\mathrm{X}$ coordinate, will be read out by one front-end block for each sensor. The single front-end block will be composed by 8 front-end chips and will have a total of 256 inputs and 256 outputs. The silicon microstrips on the bottom layer, measuring the $\mathrm{Y}$ coordinate, will be read-out by two front-end blocks, each one reading a couple of sensors with the corresponding strips shorted together through wire bondings. The read-out for the Y coordinate was spitted in two in order to limit the capacitive load of the read-out channels of the front-end chips.

The 256 outputs of each front-end block will be continuously sampled by a Xilinx Spartan 6 FPGA chip (called 'Spartan slave') so that the events can be acquired in pre-trigger mode. Another FPGA chip of the same type (called 'Spartan master') will collect the data from all the 6 read-out blocks of the plane in the event of a trigger, preprocess the data and send them to the central acquisition unit composed by a commercial Xilinx evaluation board (ML605) equipped with a Virtex 6 chip. The digital data communication between Spartan slave and master chips and between Spartan master chips and central unit will be performed through serial lines with a $200 \mathrm{Mbit} / \mathrm{s}$ speed 
capability. The central unit will correlate the data from the 4 planes, store them in a local 2 GByte RAM memory and send them to a PC through a Ethernet interface.

\section{CALORIMETER}

Starting from the results of YAG:Ce crystals characterization [21] in the previous pCT prototype, the calorimeter for residual proton energy measurement of the new pCT system, is made with an array of these crystals optically separated, each coupled with a commercial photodiode.

The calorimeter must overlap the tracker area to fully cover the entire field of view, so it consists of $2 \times 710 \mathrm{~cm}$ long crystals, each with a $3 \times 3 \mathrm{~cm}^{2}$ cross-section.

Each electronic readout channel consists of a fast charge sensitive amplifier followed by a shaper with $1 \mu$ s shaping time. The 14 analog outputs are sampled by commercial flash ADC with an on-board FPGA (National Instruments equipment).

When a proton hits one of the crystals, the 32-channels Digitizer Adapter Module (NI 5751), generates a trigger signal, to start data acquisition, and an event label (GENGlobal Event Number), to synchronize data from the different tracker planes and from the calorimeter. This module communicates to tracker Central Unit: it receives veto signal and it sends trigger signal and GEN.

The FlexRIO module includes a Virtex-5 SX50T FPGA, programmable with LabVIEW, providing data pre-elaboration. In order to reduce data to store, for each analog signal only its amplitude, proportional to the particle energy, is saved.

Finally, using a $1.9 \mathrm{GHz}$ Intel Celeron T3100 Dual Core (NI PXIe-8102 RT), the calorimeter data is stored and transferred by Ethernet connection to DAQ- PC.

\section{CONCLUSIONS}

A first prototype for $\mathrm{pCT}$, based on a silicon tracker and a YAG:Ce calorimeter, with $5 \times 5 \mathrm{~cm}^{2}$ active area has been developed and tested with proton beams in the framework of the PRIMA collaboration. An upgrade of the prototype, based on the same technology for the detectors, is under development in order to face the challenges related to the clinical application. In particular the active area has been enlarged to $5 \times 20 \mathrm{~cm}^{2}$ in order to be able to image objects of clinical interest. Moreover the data acquisition system is being optimized so that the time required to acquire a tomography data set would be of the order of a minute, compatible with other imaging techniques. The effect of MCS inside the silicon sensors has been evaluated by simulation allowing for the optimal choice of the sensors thickness. A new front-end chip has been designed to improve the performances of the silicon sensors read-out in terms of noise and channel uniformity. A larger area YAG:Ce calorimeter with novel electronics is under development.

\section{REFERENCES}

[1] U. Schneider, E. Pedroni, and A. Lomax, "The calibration of CT Hounsfield units for radiotherapy treatment planning," Phys. Med. Biol., vol. 41, pp. 111-124, 1996.

[2] A. A. M. Mustafa and D. F. Jackson, Phys. Med. Biol., 26-3, pp. 461472, 1981.
[3] D. C. Williams, Phys. Med. Biol., 49, pp. 2899-2911, 2004.

[4] T. Li et al., Med. Phys., 33-3, pp. 699-706, 2006.

[5] M. Bruzzi et al. (2007). Prototype tracking studies for proton CT. IEEE Trans. Nucl. Sci., vol. 54, p. $140-145$.

[6] G.Cirrone et al. (2007) Monte Carlo Studies of a Proton Computed Tomography System. IEEE Trans. Nucl. Sci., vol. 54, p. 1487-1491, ISSN: 0018-9499

[7] C. Talamonti et al. (2010). Proton radiography for clinical applications. Nucl.Inst.Meth. A vol. 612, p. 571-575

[8] G.A.P. Cirrone et al. (2007). The Italian project for a proton imaging device. Nucl.Inst.Meth. A vol. 576, p. 194-197.

[9] D. Menichelli et al., "Characterization of a Silicon Strip Detector and a YAG:Ce Calorimeter for a Proton Computed Radiography Apparatus", IEEE Trans. Nucl. Sci., vol. 57, no. 1, (2010) 8-16.

[10] C. Civinini et al., "Towards a proton imaging system", Nucl. Instr. Meth. A, vol. 623, Issue I, pp. 588-590, Nov. 2010.

[11] V. Sipala et al. (2010). A proton imaging device: Design and status of realization. Nucl.Inst.Meth. A vol. 612, p. 566-570.

[12] GAP.Cirrone et al., Monte Carlo evaluation of the Filtered Back Projection method for image reconstruction in proton computed tomography, Nucl.Inst.Meth. A vol. 658, p. 78-83,(2011).

[13] V. Sipala et al., "PRIMA: an apparatus for medical application", Nucl. Instr. Meth. A, vol. 658, Issue I, pp. 73-77, Dec. 2011.

[14] V. Sipala et al., Tomographic images by proton Computed Tomography system for proton therapy applications, 2011 IEEE Nuclear Science Symposium Conference Record. p. 3505-3507, ISBN: 9781467301206, doi: 10.1109/NSSMIC.2011.6152644

[15] C. Talamonti et al., PRIMA Proton Imaging for Clinical Application, 2012 IEEE Nuclear Science Symposium Conference Record.

[16] M. Bruzzi et al., The PRIMA (Proton Imaging) collaboration: Status of the development of a proton Computed Tomography Scanner, 2012 IEEE Nuclear Science Symposium Conference Record.

[17] E. Vanzi et al., "The PRIMA collaboration: preliminary results in FBP reconstruction of pCT data", presented at RESMDD12 Conference, Florence, October 9-12, 2012, submitted to Nucl. Instrum. Meth A.

[18] M. Scaringella et al., The PRIMA (Proton Imaging) collaboration: development of a proton Computed Tomography apparatus, presented at RESMDD12 Conference, Florence, October 9-12, 2012, submitted to Nucl. Instrum. Meth A

[19] M. Schulte et al. Med. Phys. 35 (11), pag. 4849, 2008.

[20] J. Beringer et al. (Particle Data Group), Phys. Rev. D86, 010001 (2012)

[21] V. Sipala et al. "YAG(Ce) crystal characterization with proton beams", Nucl. Instr. And Meth. A 654 (2011) 349-353. 